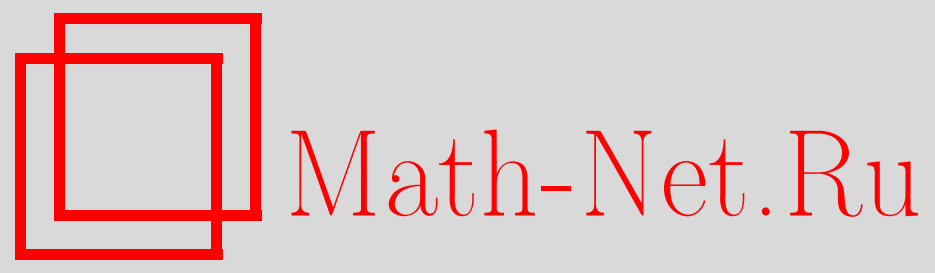

А. П. Баранов, Ю. А. Баранов, Аппроксимация моментов произвольных целых порядков обобщенными факториальными степенями, Дискрет. матем., 2005, том 17, выпуск 1, 50-67

DOI: https://doi.org/10.4213/dm87

Использование Общероссийского математического портала Math-Net.Ru подразумевает, что вы прочитали и согласны с пользовательским соглашением http: //www . mathnet.ru/rus/agreement

Параметры загрузки:

IP : 54.92 .164 .108

26 апреля 2023 г., 14:09:13

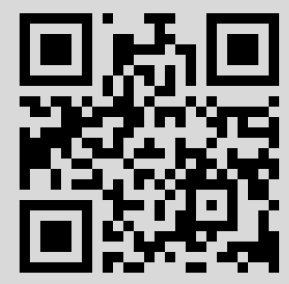




\title{
Аппроксимация моментов произвольных целых порядков обобщенными факториальными степенями
}

\author{
() 2005 г. А. П. Баранов, Ю. А. Баранов
}

Для неотрицательных целочисленных случайных величин $\xi$ рассматривается возможность построения аппроксимаций моментов $E \xi^{m}$, где $m$ - целые, в том числе отрицательные, величины. Находится оценка разности

$$
\mathbf{E} \xi^{m}-\sum_{k=0}^{5}\left\{\begin{array}{c}
m \\
m-k
\end{array}\right\} \mathbf{E} \xi^{\frac{m-k}{}}
$$

где величины $\left\{\begin{array}{c}m \\ m-k\end{array}\right\}$ являются продолжениями на все целые величины $m$ чисел Стирлинга 2-го рода, а функции $x^{\underline{m}}-$ обобщенными факториальными степенями.

При рассмотрении распределений статистик мощности рассеивания, введенных в [2], в частности, для решения задач принадлежности наблюдений заданному распределению, возникает необходимость изучения моментов целочисленных случайных величин $\xi$ как для положительных, так и для отрицательных степеней $m$. Для положительных $m$ эта проблема разрешается использованием известного представления степеней функции $x^{m}$ в виде разложения по факториальным степеням с числами Стирлинга 2-го рода, которые мы, следуя [3], будем обозначать через $\left\{\begin{array}{c}m \\ k\end{array}\right\}$, то есть

$$
x^{m}=\sum_{k=0}^{m}\left\{\begin{array}{l}
m \\
k
\end{array}\right\} x^{\underline{k}},
$$

где

$$
x^{\underline{k}}=\frac{x !}{(x-k) !}=x(x-1) \ldots(x-k+1) .
$$

Применяя равенство (1), получаем для целых неотрицательных $m$ разложение

$$
\mathbf{E} \xi^{m}=\sum_{k=0}^{m}\left\{\begin{array}{l}
m \\
k
\end{array}\right\} \mathbf{E} \xi^{\underline{k}}=\left.\sum_{k=0}^{m}\left\{\begin{array}{l}
m \\
k
\end{array}\right\} \frac{d^{k}}{d t^{k}} \Phi(t)\right|_{t=1},
$$

где $\Phi(t)=\mathbf{E} t^{\xi}$ 
Аналогичный подход хотелось бы применить и при вычислении $\xi^{m}$ для отрицательных $m$. Представление функции $x^{m}$ для произвольных $m$ получено в [1] в виде ряда

$$
x^{m}=\sum_{k=0}^{\infty}\left\{\begin{array}{c}
m \\
m-k
\end{array}\right\} x^{\frac{m-k}{2}}, \quad x \neq 0
$$

где $\left\{\begin{array}{c}m \\ k\end{array}\right\}$ - продолжения чисел Стирлинга 2-го рода, в том числе на отрицательную область значений параметров $m, k$, а $x^{k}$ по-прежнему определяется по формуле (2) с определением факториала через стандартную гамма-функцию $\Gamma(x)$ в виде

$$
x !=\Gamma(x+1), \quad x \geqslant 0 .
$$

Мы не будем здесь углубляться в области определения чисел $\left\{\begin{array}{l}m \\ k\end{array}\right\}$ и ряда (3), сославшись на [1] и [3]. Нам будет важно, что числа $\left\{\begin{array}{c}m \\ k\end{array}\right\}$ удовлетворяют рекуррентному равенству

$$
\left\{\begin{array}{l}
m \\
k
\end{array}\right\}=\left\{\begin{array}{c}
m-1 \\
k-1
\end{array}\right\}+k\left\{\begin{array}{c}
m-1 \\
k
\end{array}\right\}
$$

для любых целых $k, m, k<m$, и

$$
\begin{aligned}
& \left\{\begin{array}{c}
m \\
0
\end{array}\right\}=0, \quad m \neq 0, \\
& \left\{\begin{array}{l}
m \\
m
\end{array}\right\}=1, \quad m=0, \pm 1, \pm 2, \ldots, \\
& \left\{\begin{array}{c}
-1 \\
k
\end{array}\right\}=(-k-1) !, \quad k=-1,-2, \ldots
\end{aligned}
$$

Определим $m$-кратный оператор $\delta_{t}^{m} \Phi(t)$ над функцией $\Phi(t)$ действительного аргумента $t$ при $m<0$ как $m$-кратный интеграл

$$
\delta_{t}^{m} \Phi(t)=\int_{0}^{t} \int_{0}^{t_{1}} \ldots \int_{0}^{t_{-m-1}} \Phi\left(t_{-m}\right) d t_{-m} \ldots d t_{1} .
$$

Везде далее мы будем рассматривать только целые $m$, и очевидно, что

$$
\mathbf{E} \xi^{\underline{m}}=\left.\delta_{t}^{m} \Phi(t)\right|_{t=1}
$$

как для отрицательного $m$, так и для положительного $m$, если полагать что

$$
\delta_{t}^{m} \Phi(t)=\frac{d^{m}}{d t^{m}} \Phi(t)
$$

когда $m \geqslant 0$.

Свойство (5) делает привлекательным применение равенства (3). Однако при этом появляется несколько трудностей.

Во-первых, $\mathbf{E} \xi^{m}$ не определено для отрицательных $m$, если $\mathbf{P}(\xi=0)>0$. Мы везде далее будем полагать, что $0^{m}=0$ для любых целых $m$, включая точку $m=0$. Это определение расходится с обсуждаемыми свойствами степенных функций в точке 0 (см. $[1,3])$, где предлагается определять $x^{0}=1$ для любых $x$. Для наших целей рассмотрения 
аппроксимации функций $x^{m}$ в точках целых значений аргумента $x$ такое различие не будет существенно, однако, как мы видим, необходимо для определенности $\mathbf{E} \xi^{m}$ при $m<0$.

Во-вторых, аппроксимация рядом (3) весьма затруднительна из-за отсутствия явного представления чисел $\left\{\begin{array}{c}m \\ k\end{array}\right\}$. Имеется лишь возможность вычисления нескольких выражений, например,

$$
\begin{aligned}
\left\{\begin{array}{l}
m \\
m
\end{array}\right\} & =1, \\
\left\{\begin{array}{c}
m \\
m-1
\end{array}\right\} & =\sum_{k=1}^{m}(k-1)=\left(\begin{array}{c}
m \\
2
\end{array}\right), \\
\left\{\begin{array}{c}
m \\
m-2
\end{array}\right\} & =\sum_{k=1}^{m}(k-2)\left(\begin{array}{c}
k-1 \\
2
\end{array}\right)=\left(\begin{array}{c}
m \\
3
\end{array}\right) \frac{3 m-5}{4}, \\
\left\{\begin{array}{c}
m \\
m-2
\end{array}\right\} & =\sum_{k=1}^{m}(k-3)\left(\begin{array}{c}
k-1 \\
2
\end{array}\right) \frac{3 k-2}{4}=\left(\begin{array}{c}
m \\
4
\end{array}\right) \frac{(m-2)(m-3)}{2}, \quad m=0,1,2 \ldots,
\end{aligned}
$$

которые далее также могут быть продолжены с помощью рекуррентного соотношения (4), но которые не позволяют получить обозримого явного выражения для чисел $\left\{\begin{array}{c}m \\ m-k\end{array}\right\}$ как функция от $m$ и $k$. Вместе с тем, непосредственно можно убедиться, что равенства (6) для $m=-1,-2, \ldots$ также дают числа, удовлетворяющие рекуррентному соотношению (4) и, следовательно, также относятся к продолжению чисел Стирлинга 2-го рода, введенному в [1]. Таким образом, имеется лишь возможность вычисления, то есть явного получения конечных сумм

$$
S_{s}(m)=\sum_{k=0}^{s}\left\{\begin{array}{c}
m \\
m-k
\end{array}\right\} \mathbf{E} \xi \frac{m-k}{s}=\left.\sum_{k=0}^{s}\left\{\begin{array}{c}
m \\
m-k
\end{array}\right\} \delta_{t}^{m-k} \Phi(t)\right|_{t=1} .
$$

Отсюда, при фиксированных $s=1,2, \ldots$ возникает задача оценки для $m<0$ разности

$$
R_{s}(m)=\mathbf{E} \xi^{m}-S_{s}(m)+\sum_{k=0}^{s}\left\{\begin{array}{c}
m \\
m-k
\end{array}\right\} \frac{\mathbf{P}(\xi=0)}{(k-m) !} .
$$

Мы будем решать эту задачу, получив сначала явное выражение для разности

$$
R_{s}(m, x)=x^{m}-S_{s}(m, x),
$$

где

$$
S_{s}(m, x)=\sum_{k=0}^{s}\left\{\begin{array}{c}
m \\
m-k
\end{array}\right\} x \underline{m-k} .
$$

Предварительно рассмотрим один полезный пример, полагая $m=-1$ и $\xi \sim \Pi(\alpha)$, то есть, что случайная величина $\xi$ распределена по закону Пуассона со средним $\alpha$.

Как отмечается в [1] на стр. 413, еще в 1730 году Стирлингом получена формула

$$
\begin{aligned}
\frac{1}{x} & =\sum_{k=1}^{s+1} \frac{(k-1) !}{(x+1) \ldots(x+k)}+\frac{(s+1) !}{x(x+1) \ldots(x+s+1)} \\
& =\sum_{k=0}^{s}\left\{\begin{array}{c}
-1 \\
-1-k
\end{array}\right\} x \frac{-k-1}{x}+\frac{(s+1) !}{x} x \frac{-s-1}{}, \quad x \neq 0 .
\end{aligned}
$$


Тем самым

$$
R_{s}(-1, x)=\frac{(s+1) !}{x} x-s-1 .
$$

Используя (5), легко убедиться, что

$$
\mathbf{E} \xi^{-k}=\alpha^{-k} \mathbf{P}(\xi \geqslant k), \quad k=1,2, \ldots,
$$

поэтому

$$
\begin{aligned}
S_{s}(-1) & =\mathbf{E} S_{s}(-1, \xi)=\sum_{k=0}^{s} k ! \alpha^{-k-1} \mathbf{P}(\xi \geqslant k+1), \\
\mathbf{E} \xi^{-1} & =\sum_{k=0}^{s} k !\left(\alpha^{-k-1} \mathbf{P}(\xi \geqslant k+1)-e^{-\alpha} /(k+1) !\right)+(s+1) ! \mathbf{E}\left(\xi^{-1} \xi \frac{-s-1}{2}\right) .
\end{aligned}
$$

Отметим, что (см. [4], стр. 602)

$$
\begin{aligned}
S_{s}(-1)-e^{-\alpha} \sum_{k=0}^{s} \frac{1}{k+1} & =\sum_{k=0}^{s} k ! \alpha^{-k-1} \mathbf{P}(\xi \geqslant k+2) \\
& =e^{-\alpha}\left(\sum_{k=0}^{s} \frac{\alpha}{(k+1)(k+2)}+\frac{\alpha^{2}}{(k+1)(k+2)(k+3)} \cdots\right) \\
& =e^{-\alpha} \sum_{j=1}^{\infty} \frac{\alpha^{j}}{j}\left(\frac{1}{j !}-\frac{1}{(s+2) \ldots(s+j+1)}\right) \leqslant 1,
\end{aligned}
$$

и последняя оценка имеет место при любых $s$.

Отметим также, что при фиксированном $s$ и $\alpha \rightarrow \infty$ из (13) вытекает оценка

$$
S_{s}(-1)-e^{-\alpha} \sum_{k=0}^{s} \frac{1}{k+1}=\sum_{k=0}^{s} k ! \alpha^{-k-1}+O\left(e^{-\alpha}\right) .
$$

Теперь видно, что для получения асимптотического при $\alpha \rightarrow \infty$ разложения $\mathbf{E} \xi^{-1}$ в (12)

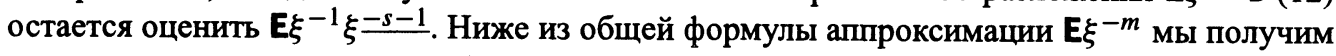
и оценку остаточного члена в (12).

Лемма 1. Для челых отричательньх $m u$ чельх $s \geqslant 0$ разность $R_{s}(m, x)$ при $x \neq 0,-1, \ldots, m-s$ имеет вид

$$
R_{s}(m, x)=\sum_{r=0}^{-m-1} \frac{s-r-m}{x^{r+1}} x \underline{r+m-s}\left\{\begin{array}{c}
r+m \\
r+m-s
\end{array}\right\} .
$$

Доказательство. Пусть

$$
\delta_{s}(m, x)=R_{s}(m, x)-\frac{1}{x} R_{s}(m+1, x) .
$$

Тогда имеет место очевидное равенство

$$
R_{s}(m, x)=\delta_{s}(m, x)+\frac{1}{x} \delta_{s}(m+1, x)+\ldots+x^{m+1} \delta_{s}(-1, x),
$$


в правой части которого $-m$ слагаемых так же, как и в первой части доказываемого равенства (14). Непосредственно из формулы (8) вытекает равенство

$$
\left.\delta_{s}(m, x)=\sum_{k=0}^{s} x^{-1} x \frac{m-k}{x(m-k+1)}\left\{\begin{array}{c}
m \\
m-k+1
\end{array}\right\}-(m-k)\left\{\begin{array}{c}
m+1 \\
m+1-k
\end{array}\right\}\right)
$$

и, если справедливо (14), то

$$
\delta_{s}(m, x)=x^{-1} x \frac{m-s}{s-m)}\left\{\begin{array}{c}
m \\
m-s
\end{array}\right\} .
$$

Докажем справедливость (17) индукщией по $s$, полагая $m$ любым целым отрицательным числом и $x \neq 0,-1, \ldots, m-s, \ldots$ Непосредственно из (16) следует, что

$$
\delta_{0}(m, x)=R_{0}(m, x)-\frac{1}{x} R_{0}(m+1, x)=(-m) x^{-1} x^{\underline{m}},
$$

и это выражение соответствует (17) при $s=0$, если учесть имеющее место для любых целых $m$ равенство

$$
\left\{\begin{array}{c}
m \\
m+1
\end{array}\right\}=0 \text {. }
$$

Отметим, что равенство для $\delta_{0}(-1, x)$ совпадает с равенством $(10)$, если иметь в виду, что $R_{0}(0, x)=0$.

Используя (16), представим $\delta_{s+1}(m, x)$ в виде

$$
\left.\delta_{s+1}(m, x)=\delta_{s}(m, x)+x^{-1} x \frac{m-s-1}{x(m-s)}\left(\begin{array}{c}
m \\
m-s
\end{array}\right)-(m-s-1)\left\{\begin{array}{l}
m+1 \\
m-s
\end{array}\right\}\right) .
$$

В последнее равенство подставим выражение для $\delta_{s}(m, x)$, соответствующее предположению (17), и применим рекуррентное соотношение (4) в виде

$$
\left\{\begin{array}{l}
m+1 \\
m-s
\end{array}\right\}=\left\{\begin{array}{c}
m \\
m-s-1
\end{array}\right\}+(m-s)\left\{\begin{array}{c}
m \\
m-s
\end{array}\right\}
$$

Проводя приведение подобных членов, получаем, что

$$
\begin{aligned}
& \delta_{s+1}(m, x)=x^{-1}\left(x \underline{m-s}(s-m)\left\{\begin{array}{c}
m \\
m-s
\end{array}\right\}+x(m-s) x^{\frac{m-s-1}{2}}\left\{\begin{array}{c}
m \\
m-s
\end{array}\right\}\right) \\
& +x^{-1} x \frac{m-s-1}{m+1}\left\{\begin{array}{l}
m \\
m-s
\end{array}\right\}(s+1-m)
\end{aligned}
$$

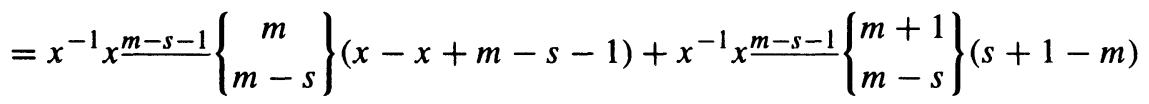

$$
\begin{aligned}
& =x^{-1} x \frac{m+s-1}{s+1-m)}\left(\left\{\begin{array}{l}
m+1 \\
m-1
\end{array}\right\}-(m-s)\left\{\begin{array}{c}
m \\
m-s
\end{array}\right\}\right) \\
& =x^{-1} x \frac{m-s-1}{(s+1-m)}\left\{\begin{array}{c}
m \\
m-s-1
\end{array}\right\} \text {. }
\end{aligned}
$$

Последнее выражение соответствует $\delta_{s+1}(m, x)$, вычисленному по формуле (17), что и заканчивает индуктивное доказательство леммы. 
Лемма 1 продолжает формулу Стирлинга (9) на функции $x^{m}, m=-1,-2, \ldots$, поскольку (10) является частным случаем (14) при $m=-1$.

Нашей целью является аппроксимация моментов целочисленных случайных величин отрицательных и положительных порядков. Этой цели служит следующая теорема.

Теорема 1. Для любых иельх $m, s, s \geqslant 0$, и положительных иелых х существует постоянная $C(m, s)$, зависящая только от $m$ и $s$, при которой имеет место неравенство

$$
\left|x^{m}-\sum_{k=0}^{s}\left\{\begin{array}{c}
m \\
m-k
\end{array}\right\} x \frac{m-k}{}\right|<x^{m-s-1} C(m, s) .
$$

Доказательство. Действительно, для $m \geqslant 0$ и целых $x>0$

$$
x^{\underline{m}}=x(x-1) \ldots(x-m+1)<x^{m} .
$$

Вместе с тем, для $m<0$ и $x>0$

$$
x^{\underline{m}}=\frac{1}{(x+1) \ldots(x-m)}<x^{m} .
$$

Поэтому справедливость (18) и сушествование постоянной $C(m, s)$ вытекает для $m \geqslant 0$ из (1), а для $m<0$ из (14).

Нетрудно также видеть, что как для случая $m \geqslant 0$, так и для $m<0$, постоянная $C(m, s)$ может быть определена в, так сказать, конечном виде, с использованием либо формулы (1), либо леммы 1.

Неравенство (18), по нашему мнению, имеет один недостаток при его применении к аппроксимации момента $\mathbf{E} \xi^{m}$. Остаточный член аппроксимации

$$
\mathbf{E} \xi^{m} \approx \sum_{k=0}^{s}\left\{\begin{array}{c}
m \\
m-k
\end{array}\right\} \mathbf{E} \xi^{\frac{m-k}{2}}
$$

имеет форму $O\left(\mathbf{E} \xi^{m-s-1}\right)$, и опять для оценки точности аппроксимации требуется оценка нефакториальных моментов, тогда как факториальные моменты можно эффективно вычислять посредством взятия оператора $\delta_{t}^{m} \Phi(t)$, как это хорошо видно на примере закона Пуассона и формулы (11). Исправлению этого недостатка служит теорема 2.

Теорема 2. Для любых чельх $m u s, s \geqslant 0, u$ положительных иельх $x$ существует постоянная $C_{1}(m, s)$, зависящие только от $m$ и $s$, при которой имеет место неравенство

$$
\left|x^{m}-\sum_{k=0}^{s}\left\{\begin{array}{c}
m \\
m-k
\end{array}\right\} x \frac{m-k}{}\right|<C_{1}(m, s)\left(x \frac{m-s-1}{2}+I(x<m-s-1)\right)
$$

(здесь $I(A)-$ индикатор события $A$ ).

Доказательство. В теореме 1 мы использовали неравенство $x^{\underline{m}}<x^{m}$ для целых $m$ и $x$, $x>0$. Теперь мы используем другое неравенство для факториальной степенной функции от целочисленного положительного аргумента

$$
x^{m} \leqslant C(m)\left(x^{\underline{m}}+I(x<m)\right),
$$


где $C(m)$ - постоянная, зависящая только от $m, m$ и $x$ - целые числа, $x>0$.

Действительно, для $m=0$, очевидно, (20) выполнено при $C(0)=1$. Пусть $m>0$. Тогда достаточно положить $C(m)=m^{m}$. Действительно, при $0<x<m$ функция $x^{m}<m^{m}$, а при $x \geqslant m$ функция $x<x m-k m, k=0, \ldots, m-1$.

Поэтому

$$
\begin{aligned}
x^{m} & <x m(x m-m) \ldots\left(x m-m^{2}+m\right)+m I(x<m) \\
& =m^{m}(x(x-1) \ldots(x+m-1)+I(x<m)) .
\end{aligned}
$$

Пусть $m<0$. Тогда достаточно выбрать $C(m)=(1-m)^{-m}$, поскольку при $x \geqslant 1$

$$
x^{-1} \leqslant(1-m)(x-k)^{-1}, \quad k=-1, \ldots, m .
$$

Таким образом, неравенство (20) в условиях теоремы 2 доказано, и оценка (19) следует из теоремы 1. При этом достаточно положить $C_{1}(m, s)=C(m, s) C(m-s-1)$. Отметим, что и в этом случае постоянная $C_{1}(m, s)$ может быть выписана в явной форме.

Теорема 2 немедленно приводит нас к возможности применения аппроксимации (19) для вычисления положительных или отрицательных моментов $\mathbf{E}^{m}$ с учетом принятого нами ранее соглашения, что $0^{m}=0$ для всех целых $m$ :

$$
\begin{array}{r}
\left|\mathbf{E} \xi^{m}-\sum_{k=0}^{s}\left\{\begin{array}{c}
m \\
m-k
\end{array}\right\}\left(\mathbf{E} \xi^{\frac{m-k}{2}}-\frac{I(m<0)}{(k-m) !} \mathbf{P}(\xi=0)\right)\right| \\
\leqslant C_{1}(m, s)\left(\mathbf{E} \xi^{\frac{m-s-1}{2}}+\mathbf{P}(\xi<m-s-1)\right) .
\end{array}
$$

Возвращаясь к рассмотренному нами ранее примеру $\xi \sim \Pi(\alpha)$, получаем в формуле (12) оценку остаточного члена

$$
\left|\mathbf{E} \xi^{-1}-\sum_{k=0}^{s} k ! \alpha^{-k-1} \mathbf{P}(\xi \geqslant k+2)\right| \leqslant C_{1}(-1, s) \alpha^{-s-2} \mathbf{P}(\xi \geqslant s+2),
$$

которая при $\alpha \rightarrow \infty$ может быть приведена к виду

$$
\mathbf{E} \xi^{-1}=\sum_{k=0}^{s} k ! \alpha^{-k-1}+O\left(\alpha^{-s-2}\right) .
$$

В качестве дальнейшей иллюстрации формулы (21) отметим, что при условии $\alpha \rightarrow \infty$, $\xi \sim \Pi(\alpha)$ для любого фиксированного целого $m$

$$
\mathbf{E} \xi^{m}=\sum_{k=0}^{s}\left\{\begin{array}{c}
m \\
m-k
\end{array}\right\} \alpha^{m-k}+O\left(\alpha^{m-s-1}\right)
$$

Рассмотрим теперь возможность применения изложенной выше идеи аппроксимации моментов $\mathbf{\xi}^{m}$ факториальными моментами $\mathbf{\xi}^{\underline{k}} \underline{\mathrm{k}}$ аппроксимации смешанных моментов вида

$$
\mu_{m, \bar{k}}=\mathbf{E} \xi^{m} \prod_{i=1}^{r} \xi_{i}^{k_{i}}
$$


где $\xi_{1}, \ldots, \xi_{r}$ - независимые неотрицательные целочисленные случайные величины, $\xi=\xi_{1}+\ldots+\xi_{r}$, а параметры $m, k_{1}, \ldots, k_{r}$ суть целые числа, $k=k_{1}+\ldots+k_{r}$, $\bar{k}=\left(k_{1}, \ldots, k_{r}\right)$. По-прежнему, для определенности, будем полагать, что $0^{m}=0$ для любого $m$. Воспользуемся формулой (19) из теоремы 2 для построения аппроксимации функции

$$
f(\bar{x})=x^{m} \prod_{i=1}^{r} x_{i}^{k_{i}}
$$

от целочисленных аргументов $x_{1}, \ldots, x_{r} \geqslant 0, \bar{x}=\left(x_{1}, \ldots, x_{r}\right), x=x_{1}+\ldots+x_{r}$. Пусть

$$
\begin{aligned}
& M(\bar{x})=\left(\sum_{j=0}^{s}\left\{\begin{array}{c}
m \\
m-j
\end{array}\right\} x \frac{m-j}{}\right) \prod_{i=1}^{r} \sum_{j=0}^{s}\left\{\begin{array}{c}
k_{i} \\
k_{i}-j
\end{array}\right\} x_{i}^{k_{i}-j}, \\
& R(\bar{x})=x^{m} \prod_{i=1}^{r} x_{i}^{k_{i}}-M(\bar{x}) .
\end{aligned}
$$

Тогда

$$
M(\bar{x})=\sum_{0 \leqslant j_{0}<\ldots<j_{r} \leqslant s} x^{\frac{m-j_{0}}{x_{1}}} x_{1}^{\frac{k_{1}-j_{1}}{1}} \ldots x_{r}^{\frac{k_{r}-j_{r}}{r}}\left\{\begin{array}{c}
m \\
m-j_{0}
\end{array}\right\} \prod_{i=1}^{r}\left\{\begin{array}{c}
k_{i} \\
k_{i}-j_{i}
\end{array}\right\},
$$

и для $R(\bar{x})$ справедливо представление

$$
\begin{aligned}
R(\bar{x}) & =\prod_{j=0}^{r}\left(a_{j}+b_{j}+I_{j}\right)-\prod_{j=0}^{r} a_{j} \\
& =I_{0} I_{1} \ldots I_{r}+\sum_{n=0}^{r-1} \sum_{0 \leqslant j_{0}<\ldots<j_{n} \leqslant r} I_{j_{0}} \ldots I_{j_{n}} \prod_{i \notin\left\{j_{0}, \ldots, j_{n}\right\}}\left(a_{i}+b_{i}\right)+a_{j_{0}} \ldots a_{j_{n}} \prod_{i \notin\left\{j_{0}, \ldots, j_{n}\right\}} b_{i},
\end{aligned}
$$

где

$$
\begin{aligned}
& a_{0}=\sum_{j=0}^{s} x \frac{m-j}{,}, \quad b_{0}=x^{m-s-1}, \quad I_{0}=I(x<m-s-1), \\
& a_{i}=\sum_{j=0}^{s} x \frac{k_{i}-j}{}, \quad b_{i}=x_{i}^{k_{i}-s-1}, \quad I_{i}=I\left(x_{i}, k-s-1\right), \quad i=1, \ldots, r
\end{aligned}
$$

Нетрудно видеть, что сумма факториальных степеней всех аргументов $x, x_{1}, \ldots, x_{r}$ в произведениях вида $a_{j_{1}} \ldots a_{j_{n}} \prod_{i \notin\left(j_{1}, \ldots, j_{n}\right)} b_{i}$ после перемножения в них сумм $a_{j_{1}}, \ldots, a_{j_{n}}$ не превосходят $m-s-1+\sum_{j=1}^{r} k_{j}$ при $0 \leqslant n \leqslant r-1$.

Во всех остальных слагаемых в представлении $R(\bar{x})$ в качестве сомножителя присутствует индикаторная функция. Эти слагаемые при всех целых $x, x_{1}, \ldots, x_{r} \geqslant 0$ допускают оценки выражениями $x^{\underline{y}} x_{1}^{\underline{y_{1}}} \ldots x_{r}^{\underline{y_{r}}} I\left(x_{i}<a\right), i=1, \ldots, r$, или $x^{\underline{y}} x_{1}^{\underline{y_{1}}} \ldots x_{r} \underline{y}_{r}(x<a)$, где $a, y, y_{1}, \ldots, y_{r}-$ некоторые целые числа.

Заметим, что из (20) и неравенства $\left(x^{\underline{y}}\right)^{2} \leqslant x^{2 y}$, верного для любого целого $y$ и $x>0$, вытекает неравенство

$$
(x \underline{y})^{2}<C(2 m)(x \underline{2 y}+I(x<2 y),
$$

где $C(2 m)$ определено в (20). 
Последнее неравенство может быть использовано при оценке слагаемых с множителем, являющимся индика горной функцией. Например,

$$
\mathbf{E} \xi^{\underline{y}} I\left(\xi_{1}<a\right)<\left(C(2 y)\left(\mathbf{E} \xi^{2 y}+I(\xi<2 y)+\frac{I(y<0)}{(-y) !} \sqrt{\mathbf{P}\{\xi=0\}}\right) \mathbf{P}\left\{\xi_{1}<a\right\} .\right.
$$

Из определения функции $M(\bar{x})$ и оценки для $R(\bar{x})$ видно, что важную роль в аппроксимации смешанного момента $\mu_{m, \bar{k}}$ играют смешанные факториальные моменты вида

$$
\mu(m, \bar{k})=\mathbf{E} \xi^{m} \prod_{i=1}^{r} \xi_{i}^{k_{i}}
$$

Общий способ вычисления $\mu(m, \bar{k})$ базируется на применении операторов $\delta_{t}^{m}$ к производящей функции

$$
\Phi\left(z_{0}, z_{1}, \ldots, z_{r}\right)=\mathbf{E} z_{0}^{\xi} \prod_{i=1}^{r} z_{i}^{\xi_{i}}=\prod_{i=1}^{r} \mathbf{E}\left(z_{0} z_{i}\right)^{\xi_{i}}
$$

Очевидно, что

$$
\mu(m, \bar{k})=\left.\delta_{z_{0}}^{m} \delta_{z_{1}}^{k_{1}} \ldots \delta_{z_{r}}^{k_{r}} \Phi\left(z_{0}, z_{1}, \ldots, z_{r}\right)\right|_{z_{0}=z_{1}=\ldots=z_{r}=1}
$$

для сходящихся в круге $|z| \leqslant 1$ производящих функций $\Phi_{i}(z)=\mathbf{E} z^{\xi_{i}}, i=1, \ldots, r$. Поэтому

$$
\mu(m, \bar{k})=\left.\delta_{z_{0}}^{m} z_{0}^{k}\left(\left.\prod_{i=1}^{r} \delta_{z_{i}}^{k_{i}} \Phi_{i}\left(z_{i}\right)\right|_{z_{i}=z_{0}}\right)\right|_{z_{0}=1}
$$

Вычисление (23) для $m \geqslant 0$ и любых $k_{i}, i=1, \ldots, r$, не вызывает трудностей, поскольку известна формула Лейбница $m$-кратного дифференцирования

$$
\left.\frac{d^{m}}{d z^{m}} z^{k} f(z)\right|_{z=1}=\left.\sum_{j=0}^{m}\left(\begin{array}{c}
m \\
j
\end{array}\right) k^{\underline{j}} \frac{d^{m-j}}{d z^{m-j}} f(z)\right|_{z=1},
$$

если $f(z)$ - нужное число раз дифференцируемая функция.

Однако, для $m<0$ общей формулы $m$-кратного интегрирования произведения двух функций неизвестно. Поэтому далее смешанные моменты $\mu(m, \bar{k})$ мы будем вычислять с учетом конкретного вида $f(z)$ для определенных типов случайных величин $\xi_{i}$ и полагать

$$
\xi_{i} \sim \Pi\left(\alpha_{i}\right), \quad i=1, \ldots, r .
$$

Начнем рассмотрение с наиболее простого, но, как будет видно далее, весьма показательного случая $r=2, k_{2}=0$. В этом случае

$$
\bar{k}=k, \quad \mu(m, k)=\mathbf{E} \xi_{1}^{\frac{k}{k}}\left(\xi_{1}+\xi_{2}\right)^{\frac{m}{}}, \quad \Phi_{1}(z)=e^{\alpha_{1}(z-1)}, \quad \Phi_{2}(z)=e^{\alpha_{2}(z-1)} .
$$

При $k \geqslant 0$ взятие оператора $\delta_{z}^{k} \Phi_{1}(z)$ очевидно:

$$
\delta_{z}^{k} \Phi_{1}(z)=\alpha_{1}^{k} e^{\alpha_{1}(z-1)} .
$$


При $k<0$, проводя $(-k)$-кратное интегрирование, получаем, что

$$
\begin{aligned}
\delta_{z}^{k} \Phi_{1}(z) & =\int_{0}^{z} \int_{0}^{t_{1}} \cdots \int_{0}^{t_{-k-1}} \Phi\left(t_{-k}\right) d t_{-k} d t_{-k-1} \ldots d t_{1} \\
& =\alpha_{1}^{k}\left(e^{\alpha_{1}(z-1)}-\sum_{j=0}^{-k-1} \frac{\left(\alpha_{1} z\right)^{j}}{j !} e^{-\alpha_{1}}\right) .
\end{aligned}
$$

Объединяя два последних представления $\delta_{z}^{k} \Phi_{1}(z)$, полагая $\sum_{j=0}^{k}=0$ при $k<0$, получаем, что

$$
\mu(m, k)=\left.\alpha_{1}^{k} e^{-\alpha} \delta_{z}^{m} z^{k}\left(e^{\alpha_{1} z}-\sum_{j=0}^{-k-1} \frac{\left(\alpha_{1} z\right)^{j}}{j !}\right) e^{\alpha_{2} z}\right|_{z=1}
$$

где $\alpha=\alpha_{1}+\alpha_{2}$.

Исследование выражения (25) при произвольных $m, k$ и $\alpha_{1}, \alpha_{2}$ весьма затруднительно, и, как мы укажем далее, его удается гровести только для случая $m \geqslant 0, k \geqslant 0$. Поэтому мы ограничимся получением асимптотического приближения факториального момента $\mu(m, k)$ только при $\alpha=\alpha_{1}+\alpha_{2} \rightarrow \infty$.

Рассмотрим взятие оператора $\delta_{z}^{m}$ для функции

$$
f(z)=\left(e^{\alpha_{1} z}-\sum_{j=0}^{-k-1} \frac{\left(\alpha_{1} z\right)^{j}}{j !}\right) z^{k} e^{\alpha_{2} z}
$$

Теорема 3. Пусть $\alpha_{1}, \alpha_{2} \rightarrow \infty$ таким образом, что существуют постоянные $C_{1}, C_{2}$, при которых

$$
0<C_{1}<\alpha_{1} \alpha^{-1}<C_{2}<1 .
$$

Тогда для произвольных цельх $m, k, s, s \geqslant 0$, и для функции $f(z)$, определенной в (26),

$$
\left.\delta_{z}^{m} f(z)\right|_{z=1}=\sum_{i=0}^{s}\left(\begin{array}{c}
m \\
i
\end{array}\right) \alpha^{m-i} e^{\alpha} k^{\underline{i}}+O\left(\alpha^{m-s-1} e^{\alpha}\right) .
$$

Доказательство. Рассмотрим наиболее простой случай $m \geqslant 0$ при произвольном $k$. С этой целью воспользуемся равенством (24) и получим, что

$$
\begin{aligned}
\left.\frac{d^{m}}{d^{m} z} f(z)\right|_{z=1}= & \left.\sum_{i=0}^{m}\left(\begin{array}{c}
m \\
i
\end{array}\right) k^{\underline{i}} \frac{d^{m-i}}{d^{m-i} z} e^{\alpha z}\right|_{z=1}-\left.\sum_{j=0}^{-k-1} \sum_{i=0}^{m}\left(\begin{array}{c}
m \\
i
\end{array}\right)(k+j)^{\frac{i}{i}} \frac{\alpha_{1}^{j}}{j !} \frac{d^{m-i}}{d^{m-i} z} e^{\alpha_{2} z}\right|_{z=1} \\
= & e^{\alpha} \sum_{i=0}^{s}\left(\begin{array}{c}
m \\
i
\end{array}\right) k^{\underline{i}}\left(\alpha_{1}+\alpha_{2}\right)^{m-i}+O\left(\left(\alpha_{1}+\alpha_{2}\right)^{m-s-1} e^{\alpha}\right) \\
& +O\left(\alpha_{2}^{m} \alpha_{1}^{-k-1} I(k<0) e^{\alpha_{2}}\right),
\end{aligned}
$$

что соответствует (27), так как $\left(\begin{array}{c}m \\ s\end{array}\right)=0$ при $s>m$ и $\alpha_{2}<\left(1-C_{1}\right) \alpha$. Доказательство для случая $m<0$ более громоздко и проводится методом индукщии по $m$ для любого 
фиксированного $k$. Индуктивное доказательство построим для несколько более общей формулы

$$
\delta_{z}^{m} f(z)=e^{\alpha z} \sum_{i=0}^{s}\left(\begin{array}{c}
m \\
i
\end{array}\right) \alpha^{m-i} k^{\underline{i}-z^{k-i}}+O\left(\alpha^{m-s-1} e^{\alpha z}\right)+O\left(\alpha^{|k|} e^{\varepsilon z}\right),
$$

где $0<\varepsilon \leqslant z \leqslant 1, \varepsilon-$ некоторая постоянная, $1-C_{1}<\varepsilon<1$. Обычно понимаемый символ $O(\cdot)$ для функции $\Psi(\alpha, z)$ в записи $y(\alpha, z)=O(\Psi(\alpha, z))$ будет нами использоваться в смысле существования некоторой постоянной $C$, не зависящей от $\alpha$ и $z$, при которой

$$
y(\alpha, z)<C \Psi(\alpha, z)
$$

в области $\varepsilon \leqslant z \leqslant 1$ и $\alpha \rightarrow \infty$. Постоянная $C$ может зависеть от вида функции $\Psi(\alpha, z)$.

Рассмотрим случай $m=-1$, представив область интегрирования в виде двух частей

$$
\delta_{z}^{-1} f(x)-\int_{\varepsilon}^{z} f(t) d t=\int_{0}^{\varepsilon} f(t) d t=\sum_{j=\max (-k, 0)}^{\infty} \frac{\alpha_{1}^{j}}{j !} \int_{0}^{\varepsilon} t^{j+k} e^{\alpha_{2} t} d t \leqslant \varepsilon^{k} e^{\varepsilon \alpha}
$$

Тогда

$$
\int_{\varepsilon}^{z} f(t) d t-\int_{\varepsilon}^{z} t^{k} e^{\alpha t} d t=O\left(\sum_{j=0}^{-k-1} \frac{\alpha_{1}^{j}}{j !} \int_{\varepsilon}^{z} t^{j+k} e^{\alpha_{2} t} d t\right)+O\left(\alpha^{|k|} e^{\varepsilon z}\right)
$$

Теперь возьмем $s+1$ раз по частям интеграл:

$$
\begin{aligned}
\int_{\varepsilon}^{z} t^{k} e^{\alpha t} d t & =\left.\frac{t^{k}}{\alpha} e^{\alpha t}\right|_{\varepsilon} ^{z}-\frac{k}{\alpha} \int_{\varepsilon}^{z} t^{k-1} e^{\alpha t} d t=\ldots \\
& =\frac{e^{\alpha z}}{\alpha} \sum_{i=0}^{s}\left(\begin{array}{c}
-1 \\
i
\end{array}\right) \alpha^{-i} k^{i} z^{k-i}+O\left(\alpha^{-s-2} e^{\alpha z}\right)
\end{aligned}
$$

Объединяя (29), (30) и (31), получаем (28) при $m=-1$.

Предполагая справедливость (28) для $m \leqslant-1$, докажем справедливость (28) при $m-1$. Пусть

$$
S=S_{\alpha}(k, z)=\sum_{i=0}^{s}\left(\begin{array}{c}
m \\
i
\end{array}\right) \alpha^{m-i} k^{i} \int_{\varepsilon}^{z} t^{k-i} e^{\alpha t} d t
$$

Тогда

$$
\delta_{z}^{m-1} f(z)=\int_{0}^{z} \delta_{t}^{m} f(t) d t=S+O\left(\int_{0}^{\varepsilon} \delta_{t}^{m} f(t) d t\right)+O\left(\alpha^{m-s-1} \int_{\varepsilon}^{z} e^{\alpha z} d t\right)
$$

и достаточно рассмотреть $S$ с оценкой интеграла

$$
\int_{0}^{\varepsilon} \delta_{t}^{m} f(t) d t=\int_{0}^{\varepsilon} \int_{0}^{t_{1}} \ldots \int_{0}^{t_{-m-1}} f\left(t_{-m}\right) d t_{-m} \ldots d t_{1},
$$

где $0<t_{j} \leqslant \varepsilon, j=1, \ldots, t_{-m-1}$. 
Разложением в ряд Тейлора и сравнением слагаемых правой и левой частей неравенства

$$
e^{x} \cdot-\sum_{i=0}^{n} \frac{x^{i}}{i !} \leqslant x^{n+1} e^{x}
$$

легко убедиться в его справедливости при $n \geqslant 0$ и $x \geqslant 0$. Следовательно, при отрицательном $k$ из (34) при $0 \leqslant t_{-m-1} \leqslant \varepsilon$ вытекает, что

$$
\begin{aligned}
\int_{0}^{t_{-m-1}} f\left(t_{-m}\right) d t_{-m} & =\int_{0}^{t_{-m-1}}\left(e^{\alpha_{1} t_{-m}}-\sum_{i=0}^{-k-1} \frac{\left(\alpha_{1} t_{-m}\right)^{i}}{i !}\right) t_{-m}^{k} e^{\alpha_{2} t_{-m}} d t_{-m} \\
& \leqslant \int_{0}^{t_{-m-1}}\left(\alpha, t_{-m}\right)^{-k} t_{-m}^{k} e^{\alpha t_{-m}} d t_{-m}=O\left(\alpha_{1}^{-k} e^{\alpha \varepsilon}\right) .
\end{aligned}
$$

Поскольку интегрирование в (33) берется $(-m)$-раз по областям размера не более $\varepsilon$, для отрицательного $k$ и $\varepsilon \leqslant z \leqslant 1$

$$
\int_{0}^{\varepsilon} \delta_{t}^{m} f(t) d t=O\left(\alpha_{1}^{-k} e^{\alpha \varepsilon}\right)
$$

Для неотрицательного $k$ и $0<1-C_{1}<\varepsilon \leqslant z$

$$
\int_{0}^{\varepsilon} \delta_{t}^{m} f(t) d t \leqslant \int_{0}^{\varepsilon} \ldots \int_{0}^{t_{-m-1}} t_{-m}^{k} e^{\alpha t_{-m}} e d t_{-m} \ldots d t_{1} \leqslant e^{\alpha \varepsilon}-1=O\left(e^{\alpha \varepsilon}\right) .
$$

Следовательно, оценка (35) имеет место для интеграла (33) при любом целом $k$.

Используя равенства

$$
\begin{aligned}
\left(\begin{array}{c}
-1 \\
j
\end{array}\right)\left(\begin{array}{c}
m \\
i
\end{array}\right) & =(-1)^{j}(-1)^{i}\left(\begin{array}{c}
-m+i-1 \\
i
\end{array}\right), \\
k^{-}(k-i) & \underline{j}=\frac{k !}{(k-i-j) !}=k \stackrel{i+j}{ },
\end{aligned}
$$

применим формулу (31) к сумме

$$
\begin{aligned}
S & =\sum_{i=0}^{s}\left(\begin{array}{c}
m \\
i
\end{array}\right) \alpha^{m-i} k^{-i} \frac{e^{\alpha z}}{\alpha} \sum_{j=0}^{s}\left(\begin{array}{c}
-1 \\
j
\end{array}\right) \alpha^{-j}(k-i)-z^{k-i-j}+O\left(\alpha^{m-s-2} e^{\alpha z}\right) \\
& =e^{\alpha z} \sum_{i=0}^{s} \sum_{j=0}^{s}\left(\begin{array}{c}
-m+i-1 \\
i
\end{array}\right)(-1)^{i+j} \alpha^{m-i-j-1} z^{k-i-j} k^{i+j}+O\left(\alpha^{m-s-2} e^{\alpha z}\right) .
\end{aligned}
$$

Сделаем замену индексов суммирования $i+j=\gamma$ и, учитывая, что $m<0$, получим равенства

$$
\begin{aligned}
S & =e^{\alpha z} \sum_{\gamma=0}^{2 s}(-1)^{\gamma} \alpha^{m-\gamma-1} z^{\gamma} k^{\underline{\gamma}} \sum_{i=0}^{\gamma}\left(\begin{array}{c}
-m+i-1 \\
i
\end{array}\right)+O\left(\alpha^{m-s-2} e^{\alpha z}\right) \\
& =e^{\alpha z} \sum_{\gamma=0}^{s}(-1)^{\gamma} \alpha^{m-\gamma-1} z^{\gamma} k^{\underline{\gamma}}\left(\begin{array}{c}
-m+\gamma \\
\gamma
\end{array}\right)+O\left(\alpha^{m-s-2} e^{\alpha z}\right) \\
& =e^{\alpha z} \sum_{\gamma=0}^{s} \alpha^{m-\gamma-1} z^{\gamma} k^{\underline{\gamma}}\left(\begin{array}{c}
m-1 \\
\gamma
\end{array}\right)+O\left(\alpha^{m-s-2} e^{\alpha z}\right) .
\end{aligned}
$$


Последнее выражение для $S$ вместе с (35) приводит к (28) для $\delta_{z}^{m-1} f(z)$. Этим индуктивное по $m$ доказательство заканчивается.

Отметим, что равенство для неопределенных $m$-кратных интегралов действительного аргумента $z$

$$
\int \ldots \int z^{k} e^{\alpha z} d z=\frac{e^{\alpha z}}{\alpha^{m}} \sum_{i=0}^{k} \frac{(-1)^{i}}{\alpha^{i}}\left(\begin{array}{c}
i+m-1 \\
i
\end{array}\right) k^{i} z^{k-i}+c_{m-1}(z)
$$

при $m \geqslant 0, k \geqslant 0$ и произвольного полинома $c_{m-1}(z)$ степени $m-1$ получено И. В. Харламовым и сообщено автору в частной беседе. Этот результат имеет самостоятельный интерес, в значительной степени он натолкнул нас на разложение, приведенное в теоремe 3.

Следствие 1. Пусть $\alpha_{1}, \alpha_{2} \rightarrow \infty$ таким образом, что существуют постоянные $C_{1}, C_{2}$, при которых

$$
0<C_{1}<\alpha_{1}\left(\alpha_{1}+\alpha_{2}\right)^{-1}<C_{2}<0 \text {. }
$$

Тогда для произвольных $m, k, s, s \geqslant 0$, смешанные факториальные моменты могут быть аппроксимированы выражением

$$
\mu(m, k)=\alpha_{1}^{k} \alpha^{m} \sum_{i=0}^{s}\left(\begin{array}{c}
m \\
i
\end{array}\right) \alpha^{-i} k^{i}+O\left(\alpha^{m+k-s-1}\right) .
$$

Равенство (37) следует из (25) и (27).

Наличие аппроксимации факториальных моментов $\mu(m, k)$ позволяет построить аналогичную аппроксимацию обычных моментов

$$
\begin{aligned}
\mu_{m, k} & =\mathbf{E} \xi_{1}^{k}\left(\xi_{1}+\xi_{2}\right)^{m} \\
& =\sum_{e=0}^{s} \sum_{0 \leqslant i, j \leqslant 1, i+j=l}\left\{\begin{array}{c}
k \\
k-i
\end{array}\right\}\left\{\begin{array}{c}
m \\
m-j
\end{array}\right\} \mu(m-j, k-i)+O\left(\alpha^{m+k-s-1}\right),
\end{aligned}
$$

что вытекает из определения функции $M(x, y, z)$ и оценки разности $R(x, y, z)$. Вместе с тем, следует отметить, что получение явного выражения для (38) уже весьма громоздко даже для случая $r=2$. Однако, применение символьных преобразований с использованием равенств (6) и ЭВМ делает эту задачу вполне решаемой.

Мы предполагаем ниже продемонстрировать возможность применения формулы (38) для вычисления конкретных моментов при значениях $s=3$. Для $s=2, r=2, k_{2}=0$ эту 
работу удается сделать вручную, без применения ЭВМ:

$$
\begin{aligned}
\mu_{m, k}=\mu & m, k)+\left\{\begin{array}{c}
m \\
m-1
\end{array}\right\} \mu(m-1, k)+\left\{\begin{array}{c}
k \\
k-1
\end{array}\right\} \mu(m, k-1) \\
& +\left\{\begin{array}{c}
m \\
m-2
\end{array}\right\} \mu(m-2, k)+\left\{\begin{array}{c}
k \\
k-2
\end{array}\right\} \mu(m, k-2) \\
& +\left\{\begin{array}{c}
m \\
m-1
\end{array}\right\}\left\{\begin{array}{c}
k \\
k-1
\end{array}\right\} \mu(m-1, k-1)+O\left(\alpha^{k+m-3}\right) \\
= & \alpha_{1}^{k} \alpha^{m}+\alpha_{1}^{k} \alpha^{m-1} m k+\left\{\begin{array}{c}
m \\
m-1
\end{array}\right\} \alpha_{1}^{k} \alpha^{m}-1+\left\{\begin{array}{c}
k \\
k-1
\end{array}\right\} \alpha_{1}^{k} \alpha^{m} \\
& +\alpha_{1}^{k} \alpha^{m-2}\left(\begin{array}{c}
m \\
2
\end{array}\right) k(k-1)+\left\{\begin{array}{c}
m \\
m-2
\end{array}\right\} \alpha_{1}^{k} \alpha^{m-2}+\left\{\begin{array}{c}
k \\
k-2
\end{array}\right\} \alpha_{1}^{k-2} \alpha^{m} \\
& +\left(\begin{array}{l}
m \\
2
\end{array}\right)\left(\begin{array}{c}
k \\
2
\end{array}\right) \alpha_{1}^{k-1} \alpha^{m-1}+\left(\begin{array}{c}
m \\
2
\end{array}\right)(m-1) k \alpha_{1}^{k} \alpha^{m-2} \\
& +\left(\begin{array}{l}
k \\
2
\end{array}\right) m(k-1) \alpha_{1}^{k-1} \alpha^{m-1}+O\left(\alpha^{k+m-3}\right) \\
= & \alpha_{1}^{k} \alpha^{m}+\alpha_{1}^{k} \alpha^{m-1}\left(\left(\begin{array}{c}
m \\
2
\end{array}\right)+m k\right)+\left(\begin{array}{l}
k \\
2
\end{array}\right) \alpha_{1}^{k-1} \alpha^{m} \\
& +\alpha_{1}^{k} \alpha^{m-2}\left(\begin{array}{l}
m \\
2
\end{array}\right)\left(\begin{array}{l}
\left.k(k-1)+\frac{(m-2)(3 m-5)}{12}\right)+\left(\begin{array}{l}
k \\
3
\end{array}\right) \frac{3 k-5}{4} \alpha_{1}^{k-2} \alpha^{m} \\
+
\end{array}\right. \\
& \left(\begin{array}{l}
k \\
2
\end{array}\right) \alpha_{1}^{k-1} \omega^{m-1}\left(\left(\begin{array}{c}
m \\
2
\end{array}\right)+m(k-1)\right)+O\left(\alpha^{k+m-3}\right) .
\end{aligned}
$$

Нетрудно видеть, что, как и должно быть, (39) при $m=0$ или $k=0$ преврашается в формулу (22) при $s=2$.

Продолжим рассмотрение возможности аппроксимации смешанных факториальных моментов более сложного вида $\mu(m, \bar{k})$, через которые выражаются нефакториальные моменты $\mu_{m, \bar{k}}$

Пусть

$$
\varphi(z)=\prod_{i=1}^{r} f_{i}\left(\alpha_{i}, z\right)
$$

где

$$
f_{i}\left(\alpha_{i}, z\right)=z^{k_{i}}\left(e^{\alpha_{i} z}-\sum_{j=0}^{-k_{i}-1} \frac{\left(\alpha_{i} z\right)^{j}}{j !}\right), \quad i=1, \ldots, r .
$$

Тогда равенство (23) для случайных величин $\xi_{i} \sim \prod\left(\alpha_{i}\right), i=1, \ldots, r$, принимает вид

$$
\mu(m, \bar{k})=\left.\alpha_{1}^{k_{1}} \ldots \alpha_{r}^{k_{r}} e^{-\alpha} \delta_{z}^{m} \varphi(z)\right|_{z=1} .
$$

Нетрудно видеть, что, как и следовало ожидать, выражение (41) при $r=2, k_{2}=0$ сводится к (25).

Теорема 4. Пусть $\alpha_{1}, \ldots, \alpha_{r} \rightarrow \infty$ таким образом, что существуют не зависящие от $\alpha_{1}, \ldots, \alpha_{r}$ постоянные $c_{1}, c_{2}$, при которых

$$
0<c_{1}<\alpha_{i} \alpha^{-1}<c_{2}<1
$$


Тогда для произвольных челых $s, m, k_{i}, i=1, \ldots, r, s>0, u$ функций $\varphi(z)$, определенных равенством (40),

$$
\left.\delta_{z}^{m} \varphi(z)\right|_{z=1}=\sum_{i=0}^{s}\left(\begin{array}{c}
m \\
i
\end{array}\right) \alpha^{m-i} e^{\alpha} k^{i}+O\left(\alpha^{m-s-1} e^{\alpha}\right) .
$$

Доказательство. В идейном плане и во многих технических деталях доказательство теоремы 4 повторяет доказательство теоремы 3 с соответствующим усложнением записей. Мы рассмотрим лишь моменты, где появляется определенная новизна.

Случай $m \geqslant 0$ рассматривается аналогично с помощью (24).

Справедливость (42) для $m<0$ доказывается индуктивно по $m$ для произвольных $k_{1}, \ldots, k_{r}$, как следствие аппроксимации

$$
\delta_{z}^{m} \varphi(z)=e^{\alpha z} \sum_{i=0}^{s}\left(\begin{array}{c}
m \\
i
\end{array}\right) \alpha^{m-i} k^{i} z^{k-i}+O\left(\alpha^{m-s-1} e^{\alpha z}\right)+O\left(\alpha^{\max \left|k_{i}\right|} e^{\varepsilon z}\right)
$$

при $\varepsilon \leqslant z \leqslant 1$ и некоторой фиксированной величине $\varepsilon, 1-c_{1}<\varepsilon<1$.

Рассмотрим случай $m=-1$. Представим функщии $f_{i}\left(\alpha_{i}, z\right)$ в виде

$$
f_{i}\left(\alpha_{i}, z\right)=z^{k_{i}} \sum_{j=\max \left(-k_{i}, 0\right)}^{\infty} \frac{\left(\alpha_{i} z\right)^{j}}{j !}
$$

Тогда для любых фиксированных $\bar{k}$ и $s$

$$
\begin{aligned}
\delta_{z}^{-1}(\varphi(z))-\int_{\varepsilon}^{z} \varphi(t) d t & =\int_{0}^{\varepsilon} \varphi(t) d t=\int_{0}^{\varepsilon} \prod_{i=1}^{r} \sum_{j=\max \left(-k_{i}, 0\right)}^{\infty} \frac{\left(\alpha_{i} t\right)^{j}}{j !} d t \\
& =\sum_{j_{1}=\max \left(-k_{1}, 0\right)}^{\infty} \cdots \sum_{j_{2}=\max \left(-k_{r}, 0\right)}^{\infty} \frac{\alpha_{1}^{j_{1}} \ldots \alpha_{r}^{j_{r}}}{j_{1} ! \ldots j_{r} !} \int_{0}^{\varepsilon} t^{j_{1}+\ldots+j_{r}+k} d t \\
& =O\left(e^{\varepsilon \alpha}\right) .
\end{aligned}
$$

Теперь аналогично (30) для произвольных фиксированных $\bar{k}, s, z, \varepsilon \leqslant z \leqslant 1$, получаем соотношение

$$
\begin{aligned}
\int_{\varepsilon}^{z} \varphi(t) d t-\int_{\varepsilon}^{z} t^{k} e^{\alpha t} d t & =\int_{\varepsilon}^{z} t^{k} e^{\alpha t}\left(\prod_{i=1}^{r}\left(1-\sum_{j=0}^{-k_{i}-1} \frac{\left(\alpha_{i} t\right)^{j}}{j !} e^{-\alpha_{i} t}\right)-1\right) d t \\
& =O\left(\int_{\varepsilon}^{z} t^{k} e^{\alpha t} \sum_{i=1}^{r} \sum_{j=0}^{-k_{i}-1} \frac{\left(\alpha_{i} t\right)^{j}}{j !} e^{-\alpha_{i} t} d t\right)=O\left(\alpha^{\max \left|k_{i}\right|} e^{\varepsilon z}\right)
\end{aligned}
$$

Интеграл $\int_{\varepsilon}^{z} t^{k} e^{\alpha t} d t$ удовлетворяет равенству (31), что приводит к соотношению (42) при $m=-1$ и любом фиксированном $k$.

Используя соотношение (32), получаем, что

$$
\begin{aligned}
\delta_{z}^{m-1} \varphi(z) & =\int_{0}^{z} \delta_{t}^{m} \varphi(t) d t \\
& =S_{\alpha}(k, z)+O\left(\int_{0}^{\varepsilon} \delta_{t}^{m} \varphi(t) d t\right)+O\left(\alpha^{m-s-1} \int_{\varepsilon}^{z} e^{\alpha t} d t\right) .
\end{aligned}
$$


Напомним, что, в отличие от (32), $\alpha=\alpha_{1}+\ldots+\alpha_{r}$ и $k=k_{1}+\ldots+k_{r}$. Применим полученную при рассмотрении случая $m=-1$ оценку (44). Тогда для фиксированных $\varepsilon, m<0, \varepsilon \leqslant z \leqslant 1$

$$
\int_{0}^{\varepsilon} \delta_{t}^{m} \varphi(t) d t \leqslant \int_{0}^{\varepsilon} \ldots \int_{0}^{\varepsilon} \varphi\left(t_{-m}\right) d t_{-m} \ldots d t \leqslant \varepsilon^{-m+1} \int_{0}^{\varepsilon} \varphi(t) d t=O\left(\alpha^{\max \left|k_{i}\right|} e^{\varepsilon z}\right),
$$

и мы получаем оценку, аналогичную (35), хотя и использовали (44) вместо неравенства (34), как при выводе оценки (35).

Остается преобразовать сумму $S_{\alpha}(k, z)$, применяя формулу (31), как это было продемонстрировано при окончании доказательства теоремы 3. Указанные преобразования приводят к выражению (43) при $m-1$, чем и заканчивается индуктивное доказательство теоремы 4 .

Следствие 2. Пусть $\alpha_{1}, \ldots, \alpha_{r} \rightarrow \infty$ таким образом, что существуют постоянные $c_{1}$, $c_{2}$, при которых

$$
0<c_{1}<\alpha_{i} \alpha^{-1}<c_{2}<\infty, \quad i=1, \ldots, r .
$$

Тогда для произвольных фиксированных наборов иельх чисел $\bar{k}, m, s, s \geqslant 0$, смешанные факториальные моменты аппроксимируются выражением

$$
\mu(m, \bar{k})=\alpha_{1}^{k_{1}} \ldots \alpha_{r}^{k_{r}} \alpha^{m} \sum_{i=0}^{s}\left(\begin{array}{c}
m \\
i
\end{array}\right) \alpha^{-i} k^{i}+O\left(\alpha^{m+k-s-1}\right) .
$$

Аппроксимация (46) непосредственно следует из (41) и (42).

Отметим, что в условиях (45) оценка остатка $O\left(\alpha^{m+k-s-1}\right)$ по порядку малости меньше, чем наименьший из, так сказать, содержательных членов разложения (46).

Наличие аппроксимации факториальных моментов позволяет аналогично (38) построить аппроксимацию обычных моментов

$$
\mu_{m, \bar{k}}=\mathbf{E}\left(\xi_{1}+\ldots+\xi_{r}\right)^{m} \prod_{i=1}^{r} \xi_{i}^{k_{i}}=\mathbf{E} M(\bar{\xi})+\mathbf{E} R(\bar{\xi}) .
$$

Из данного ранее определения функций $M(\bar{x})$ вытекает равенство

$$
\mathbf{E} M(\bar{\xi})=\sum_{0 \leqslant j_{0}, \ldots, j_{r} \leqslant s} \mu\left(m-j_{0}, \bar{k}-\bar{j}\right)\left\{\begin{array}{c}
m \\
m-j_{0}
\end{array}\right\} \prod_{i=1}^{r}\left\{\begin{array}{c}
k_{i} \\
k_{i}-j_{i}
\end{array}\right\},
$$

где $\bar{j}=\left(j_{1}, \ldots, j_{r}\right)$.

Оценка величины $\mathbf{E} R(\bar{\xi})$ для $\xi \sim \Pi\left(\alpha_{i}\right), i=1, \ldots, r$, при ограничениях (45) имеет вид

$$
\mathbf{E} R(\bar{\xi})=O\left(\alpha^{m+k-s-1}\right),
$$

что непосредственно следует из оценки функции $R(x)$ и (46).

Следствие 3. Пусть $\alpha \rightarrow \infty u \alpha_{1}, \ldots, \alpha_{r}$ удовлетворяют (45) при $i=1, \ldots, r$. Тогда для произвольных фиксированных наборов челых чисел $\bar{k}, m, s, s \geqslant 0$, смешанные моменты аппроксимируются выражением

$$
\mu_{m . \bar{k}}=\mathbf{E} M(\bar{\xi})+O\left(\alpha^{m+k-s-1}\right),
$$

где $\alpha=\alpha_{1}+\ldots+\alpha_{r} u \mathbf{E} M(\bar{\xi})$ имеет представление (47).

3 Дискретная математика, т.17 №1 
Нетрудно видеть, что выражение (38) является частным случаем (48) при $r=1$. Ранее представление (39) для $\mu_{m, k}$ продемонстрировало техническую сложность вычисления вручную разложений для $\mu_{m, k}$. Представление (47) еще более громоздко. Однако, для его вычисления не требуется делать сколько-нибудь оригинальных вычислений. Вся проблема состоит в громоздкости проведения простых алгебраических преобразований подстановки в (47) разложений (46) и явных представлений для чисел Стирлинга 2-го рода, вычисленных, например, по формулам (6), и их продолжений. После проведения замены переменных следует провести приведение подобных членов и выписать результат в порядке убывания малости степеней $\alpha$. Тем не менее, как показал опыт получения (39), эта работа вручную возможна только при малых $s=2, r=1$ значениях параметров.

Мы поручили эту рутинную работу ЭВМ, используя пакет прикладных программ MAPLE V6. Однако, даже с применением ЭBM не удается явно выписать представление для произвольных $s$ и $r$.

Мы приведем результат описанной рутинной работы на ЭВМ с помощью пакета MAPLE V6 для $s=3, r=3$, полагая $\alpha_{i}=x_{i} \alpha, 0<x_{i}<1, i=1,2,3, x_{1}+x_{2}+x_{3}=1$. В этих условиях мы получаем разложение для $\mu_{m, k_{1}, k_{2}, k_{3}}$ как разложение по степеням $\alpha$. Приведем полученное разложение по первым трем степеням $\alpha$ :

$$
\begin{aligned}
& \mu_{m, k_{1}, k_{2}, k_{3}}=\alpha^{m+k_{1}+k_{2}+k_{3}} x_{1}^{k_{1}} x_{2}^{k_{2}} x_{3}^{k_{3}}+(1 / 2) \alpha^{m+k_{1}+k_{2}+k_{3}} x_{1}^{\left(k_{1}-1\right)} x_{2}^{\left(k_{2}-1\right)} x_{3}^{\left(k_{3}-1\right)}\left(-m x_{1} x_{2} x_{3}\right. \\
& +k_{3}^{2} x_{1} x_{2}+m^{2} x_{1} x_{2} x_{3}-k_{1} x_{2} x_{3}+2 m k_{1} x_{1} x_{2} x_{3}+2 m k_{2} x_{1} x_{2} x_{3}+2 m k_{3} x_{1} x_{2} x_{3} \\
& \left.+k_{2}^{2} x_{1} x_{3}-k_{2} x_{1} x_{3}+k_{1}^{2} x_{2} x_{3}-k_{3} x_{1} x_{2}\right) / \alpha+(1 / 24) \alpha^{m+k_{1}+k_{2}+k_{3}} x_{1}^{\left(k_{1}-2\right)} \\
& \times x_{2}^{\left(k_{2}-2\right)} x_{3}^{\left(k_{3}-2\right)}\left(-14 k_{1}^{3} x_{3}^{2} x_{2}^{2}-10 m x_{3}^{2} x_{1}^{2} x_{2}^{2}+3 m^{4} x_{3}^{2} x_{1}^{2} x_{2}^{2}+24 m^{2} k_{1} k_{2} x_{3}^{2} x_{1}^{2} x_{2}^{2}\right. \\
& +24 m^{2} k_{1} k_{3} x_{3}^{2} x_{1}^{2} x_{2}^{2}+21 k_{1}^{2} x_{3}^{2} x_{2}^{2}+24 m^{2} k_{2} k_{3} x_{3}^{2} x_{1}^{2} x_{2}^{2}-24 m k_{1} k_{2} x_{3}^{2} x_{1}^{2} x_{2}^{2} \\
& -24 m k_{1} k_{3} x_{3}^{2} x_{1}^{2} x_{2}^{2}-14 m^{3} x_{3}^{2} x_{1}^{2} x_{2}^{2}+21 m^{2} x_{3}^{2} x_{1}^{2} x_{2}^{2}+12 m^{3} k_{1} x_{3}^{2} x_{1}^{2} x_{2}^{2} \\
& +12 m^{3} k_{2} x_{3}^{2} x_{1}^{2} x_{2}^{2}+12 m^{3} k_{3} x_{3}^{2} x_{1}^{2} x_{2}^{2}+12 m^{2} k_{1}^{2} x_{3}^{2} x_{1}^{2} x_{2}^{2}+12 m^{2} k_{2}^{2} x_{3}^{2} x_{1}^{2} x_{2}^{2} \\
& +12 m^{2} k_{3}^{2} x_{3}^{2} x_{1}^{2} x_{2}^{2}-36 m^{2} k_{1} x_{3}^{2} x_{1}^{2} x_{2}^{2}-36 m^{2} k_{3} x_{3}^{2} x_{1}^{2} x_{2}^{2}-36 m^{2} k_{2} x_{3}^{2} x_{1}^{2} x_{2}^{2} \\
& -12 m k_{1}^{2} x_{3}^{2} x_{1}^{2} x_{2}^{2}-12 m k_{2}^{2} x_{3}^{2} x_{1}^{2} x_{2}^{2}-12 m k_{3}^{2} x_{3}^{2} x_{1}^{2} x_{2}^{2}+24 m k_{3} x_{3}^{2} x_{1}^{2} x_{2}^{2} \\
& +24 m k_{1} x_{3}^{2} x_{1}^{2} x_{2}^{2}-24 m k_{2} k_{3} x_{3}^{2} x_{1}^{2} x_{2}^{2}+24 m k_{2} x_{3}^{2} x_{1}^{2} x_{2}^{2}+12 m k_{1} k_{3}^{2} x_{3} x_{1}^{2} x_{2}^{2} \\
& -6 m^{2} k_{3} x_{3} x_{1}^{2} x_{2}^{2}+12 m k_{1}^{2} k_{3} x_{3}^{2} x_{1} x_{2}^{2}+18 m k_{3} x_{3} x_{1}^{2} x_{2}^{2}+12 m k_{2} k_{3}^{2} x_{3} x_{1}^{2} x_{2}^{2} \\
& -6 m^{2} k_{2} x_{3}^{2} x_{1}^{2} x_{2}-12 m k_{1} k_{2} x_{3}^{2} x_{1}^{2} x_{2}-30 m k_{2}^{2} x_{3}^{2} x_{1}^{2} x_{2}-12 m k_{1} k_{3} x_{3}^{2} x_{1} x_{2}^{2} \\
& +18 m k_{2} x_{3}^{2} x_{1}^{2} x_{2}+3 k_{1}^{4} x_{3}^{2} x_{2}^{2}+12 m k_{1} k_{2}^{2} x_{3}^{2} x_{1}^{2} x_{2}-10 k_{1} x_{3}^{2} x_{2}^{2}-12 m k_{2} k_{3} x_{3}^{2} x_{1}^{2} x_{2} \\
& +12 m k_{3}^{3} x_{3} x_{1}^{2} x_{2}^{2}+12 m k_{1}^{3} x_{3}^{2} x_{1} x_{2}^{2}+12 m k_{2}^{2} k_{3} x_{3}^{2} x_{1}^{2} x_{2}-12 m k_{2} k_{3} x_{3} x_{1}^{2} x_{2}^{2} \\
& +6 k_{1} k_{3} x_{3} x_{1} x_{2}^{2}+6 m^{2} k_{2}^{2} x_{3}^{2} x_{1}^{2} x_{2}+3 k_{3}^{4} x_{1}^{2} x_{2}^{2}-10 k_{3} x_{1}^{2} x_{2}^{2}-12 m k_{1} k_{3} x_{3} x_{1}^{2} x_{2}^{2} \\
& -12 m k_{1} k_{2} x_{3}^{2} x_{1} x_{2}^{2}-6 k_{1} k_{3}^{2} x_{3} x_{1} x_{2}^{2}-14 k_{3}^{3} x_{1}^{2} x_{2}^{2}+21 k_{3}^{2} x_{1}^{2} x_{2}^{2}-10 k_{2} x_{3}^{2} x_{1}^{2} \\
& +12 m k_{1}^{2} k_{2} x_{3}^{2} x_{1} x_{2}^{2}+6 m^{2} k_{3}^{2} x_{3} x_{1}^{2} x_{2}^{2}-30 m k_{3}^{2} x_{3} x_{1}^{2} x_{2}^{2}+12 m k_{2}^{3} x_{3}^{2} x_{1}^{2} x_{2} \\
& +21 k_{2}^{2} x_{3}^{2} x_{1}^{2}-14 k_{2}^{3} x_{3}^{2} x_{1}^{2}+6 m^{2} k_{1}^{2} x_{3}^{2} x_{1} x_{2}^{2}+3 k_{2}^{4} x_{3}^{2} x_{1}^{2}-6 m^{2} k_{1} x_{3}^{2} x_{1} x_{2}^{2} \\
& -30 m k_{1}^{2} x_{3}^{2} x_{1} x_{2}^{2}+18 m k_{1} x_{3}^{2} x_{1} x_{2}^{2}+6 k_{1}^{2} k_{2}^{2} x_{3}^{2} x_{1} x_{2}-6 k_{2}^{2} k_{3} x_{3} x_{1}^{2} x_{2} \\
& -6 k_{2} k_{3}^{2} x_{3} x_{1}^{2} x_{2}+6 k_{2} k_{3} x_{3} x_{1}^{2} x_{2}-6 k_{1} k_{2}^{2} x_{3}^{2} x_{1} x_{2}+6 k_{1}^{2} k_{3}^{2} x_{3} x_{1} x_{2}^{2}+6 k_{2}^{2} k_{3}^{2} x_{3} x_{1}^{2} x_{2} \\
& \left.-6 k_{1}^{2} k_{3} x_{3} x_{1} x_{2}^{2}+6 k_{1} k_{2} x_{3}^{2} x_{1} x_{2}-6 k_{1}^{2} k_{2} x_{3}^{2} x_{1} x_{2}\right) / \alpha^{2}+O\left(\alpha^{m+k_{1}+k_{2}+k_{3}-3}\right) \text {. }
\end{aligned}
$$

Для контроля правильности полученного выражения можно проверить его на совпа- 
дение с (39), полагая $k_{3}=k_{2}=0$.

В заключение отметим, что равенство (47) по существу не зависит от распределения случайных величин $\xi_{1}, \ldots, \xi_{r}$ и, следовательно, может быть использовано для вычисления смешанных моментов $\mu_{m, \bar{k}}$ для произвольно распределенных случайных величин, принимающих с положительной вероятностью целые неотрицательные значения. При этом для распределений случайных величин $\xi_{1}, \ldots, \xi_{r}$, отличных от закона Пуассона, потребуется получение аналогов теорем 3,4 и оценок для величины $\mathbf{E} R(\bar{\xi})$. Представляется, что подобный результат может быть получен для случайных величин $\xi_{1}, \ldots, \xi_{r}$, распределенных по биномиальному закону, отрицательному биномиальному закону, включая геометрический закон распределения, гипергеометрическому распределению, а также по ряду других законов, для которых удается в явном виде получить разложения основного интеграла в (23) вида

$$
\int_{0}^{z} t^{m} \prod_{i=1}^{r} \varphi_{i}(t) d t
$$

где $\varphi_{i}(t)$ получено как следствие применения оператора $\delta_{t}^{k_{i}}$ к производящей функщии соответствующего закона распределения. В свою очередь, главной трудностью при вычисленин оператора $\delta_{t}^{k} \Phi(t)$ является $k$-кратное вычисление интеграла

$$
\int_{0}^{t} \int_{0}^{t_{1}} \cdots \int_{0}^{t_{k-1}} \Phi\left(t_{-k}\right) d t_{-k} \ldots d t_{1},
$$

где $\Phi(t)$ - соответствующая производящая функция, $k<0$.

Для тех законов распределения, у которых производящая функция допускает явное вычисление (50) и получение разложения для (49), например, аналогичного (42), изложенный метод вычисления смешанных факториальных моментов приемлем с точки зрения получения конкретных разложений.

\section{Список литературы}

1. Read T. R. G., Gressie N. A. S., Goodness-of-fit statistics for discrete multivariate data. Springer, New York, 1988.

2. Knuth D. E., Two notes on notation. Amer. Math. Monthly (1992) 99, 403-422.

3. Грэхем Р., Кнут Д., Паташник О., Конкретная математика. Москва, Мир, 1998.

4. Прудников А. П., Брычков Ю. А., Маричев О. И., Интегралы и ряды. Москва, Наука, 1981.

Статья поступила 20.07.2004. 